\title{
SEQÜÊNCIA DO TRATAMENTO DE CURTA DURAÇÃO DA TUBERCULOSE PULMONAR EM UNIDADES SANITẢRIAS DO VALE DO PARAÍBA, 1980-1981, SÃO PAULO, BRASIL
}

\author{
Marilia Belluomini* \\ Hilda Kuniy Tagusagawa**
}

\begin{abstract}
BELLUOMINI, M. \& TAGUSAGAWA, H. K. Seqüência do tratamento de curta duração da tuberculose pulmonar em Unidades Sanitárias do Vale do Paraíba, 1980-1981, São Paulo, Brasil. Rev. Saúde públ., S. Paulo, 18:466-75, 1984.
\end{abstract}

RESUMO: Estuda-se a seqüência do tratamento de curta duração em doentes com tuberculose pulmonar, inscritos e com alta no período de outubro de 1980 a outubro de 1981 , nas Unidades Sanitárias do Departamento Regional de Saúde do Vale do Parấba - DRS-3 - da Secretaria da Saúde do Estado de São Paulo, Brasil. Os dados obtidos pela verificação dos prontuários dos doentes foram analisados sob os seguintes aspectos: distrito sanitário, idade $\mathrm{e}$ sexo do paciente, tipo de alta, esquema de tratamento, hospitalização, intolerância medicamentosa e tempo de negativação do escarro. Dos 308 doentes que constituíram a população de estudo, $254(82,5 \%)$ tiveram alta por cura, $21(6,8 \%)$ por abandono e $33(10,7 \%)$ por mudança de diagnóstico, óbito ou transferência. Os resultados confirmam a efetividade do tratamento encurtado da tuberculose pulmonar com os esquemas recomendados, apresentando na área trabalhada baixas taxas de abandono e de hospitalização e resultados bastante favoráveis quanto à negativação dos baciliferos.

UNITERMOS: Tuberculose pulmonar, tratamento: Ambulatórios, assistência. Tuberculose, quimioterapia.

\section{INTRODUÇÃO}

Antes da descoberta do bacilo da tubercu. lose em 1882 por Robert Koch, o tratamento da tuberculose era baseado em medidas empíricas quando imperavam as observações e as experiências, mais do que as teorias ${ }^{18}$.

Gradativamente métodos eram adotados e abandonados em constantes tentativas de êxito na cura do mal. Antigos métodos como o sangramento ou medicinas purgativas eram substituídos por recomendações enfáticas sobre dietas nutritivas, mudanças de clima, higiene pessoal ou uso de drogas contendo mercúrio, iodo, sais de arsênico ou ouro.

Com a descoberta do bacilo foi possivel o desenvolvimento de métodos preventi- vos ou terapêticos mais racionais, decorrentes do conhecimento da transmissibilidade da doença. $O$ tratamento continuava baseado apenas nos processos naturais de defesa e cura de que o próprio organismo dispunha, porém seguindo um regime higiênico-dietético, geralmente feito em sanatórios e auxiliados, quando necessário, por métodos artificiais ou procedimentos cirúrgicos ${ }^{2}$.

Em 1944, Waksman ${ }^{18}$ iniciou uma nova era na luta contra a tuberculose, anuncian. do a descoberta de um medicamento especí. fico para a sua cura - a estreptomicina.

A partir de então os doentes eram tratados com uma única droga e por perfodos

* Do Departamento de Epidemiologia da Faculdade de Saúde Pública da Universidade de São Paulo Av. Dr. Arnaldo, 715 - 01255 - São Paulo, SP - Brasil.

** Da Secretaria de Negócios da Saúde do Estado de São Paulo - Av. São Luiz, 99 - 01046 - São Paulo, SP - Brasil. 
BELLUOMINI, M. \& TAGUSAGAWA, H.K. Seqiiência do tratamento de curta duraçto da tuberculose pulmonar em unidades sanitárias do Vale do Pará́ba, 1980-1981, Sáo Paulo, Brasil. Rev. Saúde públ, S. Paulo, 18: 466-75, 1984 .

curtos ( 3 semanas a 6 meses), ocasionando oportunidades no aparecimento de doentes com resistência bacteriana. Com o aparecimento de novas drogas foi possível introduzir a quimioterapia associada e o tempo de tratamento foi expandido, sendo o de um ano o mais curto. Usualmente duravam dois anos, sendo que alguns médicos o prolongavam indefinidamente.

Com o sucesso alcançado pela quimiotera. pia algumas práticas anteriormente estabelecidas foram postas em cheque. Assim, o tratamento sanatorial, o repouso absoluto, as condições climáticas e as dietas alimentares passaram a ser questionadas e deixaram de ser consideradas imprescindíveis para garantir a cura do doente.

Com os conhecimentos no campo farmacológico e também devido às modificaçôes e às caracteristicas da epidemiologia da tuberculose, no decorrer dos últimos anos iniciaram-se investigações com o objetivo de determinar a ação das drogas, estabelecendo-se comparaçбes baseadas em variaçð̃es sobre extensão do tratamento, número de drogas utilizadas, doses e freqüência com que deveriam ser tomadas.

Fox e Mitchison ${ }^{5}$, em 1974, publicaram os resultados de uma investigação levada a efeito na África, no Japão e na Índia, preconizando o sucesso de regimes terapeuticos de curta duração. A partir desses resultados a maioria dos países passou a adotar, em caráter experimental, esquemas encurtados nos serviços de saúde que, em vista dos resultados favoráveis, vem sendo gradativamente incorporados às normas dos programas de tuberculose.

No Brasil o esquema com rifampicina (RFM), pirazinamida (PZA) e hidrazida (INH), tomados diariamente por um período de 6 meses, passou a ser recomendado a partir de 1979. Foi introduzido pela Portaria no $14^{4}$, contendo normas para o controle da tuberculose elaboradas por um grupo de especialistas de várias instituições nacionais de saúde, com o objetivo de aplicar com efi- ciência os conhecimentos atuais no tratamento da doença, com suas conseqüentes vantagens sob o ponto de vista epidemiológico, operativo e social.

No segundo semestre de 1980 as Unidades Sanitárias dos Departamentos Regionais da Secretaria de Negócios da Saúde do Estado de Sáo Paulo passaram a adotar aquele esquema para o tratamento da tuberculose. Precedeu esta introdução amplo treinamento do pessoal médico e auxiliar, em todos os níveis, para apresentar e discutir a fundamentação teórica que justificava a medida recomendada.

As equipes técnicas do Departamento Regional de Saúde do Vale do Pararba (DRS-3) e dos Distritos Sanitários (DS) que o compóem, DS de São José dos Campos, de Taubaté e de Guaratinguetá, sentiram que seria oportuno levantar alguns aspectos do tratamento encurtado da tuberculose pulmonar durante o primeiro ano de sua implantação nas unidades da DRS-3.

$O$ presente trabalho objetiva estudar alguns aspectos da seqüência do tratamento de curta duração da tuberculose pulmonar em unidades sanitárias do Vale do Paraíba, no período de outubro de 1980 a outubro de 1981.

\section{MATERIAL E METODOS}

Para o levantamento dos dados foi elaborado uma ficha com 21 itens*, preenchidos a partir dos prontuários dos doentes de 31 unidades sanitárias pertencentes aos distritos sanitários de São José dos Campos, Taubaté e Guaratinguetá.

Os prontuários escolhidos foram os de doentes submetidos a tratamento de tuberculose pulmonar pelo esquema curto, inscritos e com alta no período de outubro de 1980 a outubro de 1981 .

As equipes técnicas receberam um roteiro* e foram orientadas no sentido de utilizar o Boletim mensal de altas de doentes de tu. berculose - SVE-7, para identificar os pron-

* O modelo da ficha e do roteiro poderão ser fornecidos aos interessados mediante solicitaçío encaminhada aos autores. 
BELLUOMINI, M. \& TAGUSAGAWA, H.K. Sequiência do tratamento de curta duração da tuberculose pulmonar em unidades sanitárias do Vale do Paraf́ba, 1980-1981, São Paulo, Brasil. Rev. Saúde puibl, S. Paulo, 18: 466-75, 1984.

tuários e preencher as fichas dos doentes que constituíram a população de estudo.

Os prontuários foram estudados sob as seguintes variáveis: distrito sanitário, idade e sexo dos doentes, tipo de alta, esquema de tratamento, hospitalização, intolerância medicamentosa e tempo de negativação do escarro.

A população de estudo foi constiturda por 308 doentes que reuniram as condições necessárias para o objetivo proposto no trabalho.

Foi exclurda do estudo a unidade sanitária de Campos do Jordão, visto o retardo na introdução do tratamento pelo esquema curto, o que impossibilitou a alta de doentes dentro do período estabelecido.

O método diagnóstico mais utilizado foi a baciloscopia, solicitada em $55,6 \%$ associada aos Raios X e em $24,3 \%$ como método diagnóstico isolado.

\section{RESULTADOS E COMENTÁRIOS}

\section{Distrito Sanitário}

Dos 308 doentes inscritos com tuberculose pulmonar e submetidos a tratamento curto, no período estudado, $43,2 \%$ pertenciam ao DS de São José dos Campos, $32,1 \%$ ao DS de Guaratinguetá e $24,7 \%$ ao DS de Taubaté (Tabela 1). Justifica-se a maior percentagem de inscritos no DS de São José dos Campos, visto ser esta cidade atualmente um grande pólo de atração para o crescimento populacional e possuir um parque industrial em constante ascenção. 0 município de São José dos Campos congrega $42,1 \%$ da população da região, ficando $28,9 \%$ para Guaratinguetá e 29,0\% para Taubaté. Importante ressaltar que embora pertencendo ao DS de São José dos Campos, não foi incluído no estudo a unidade sanitária de Campos do Jordão. Sendo uma estância climática tradicionalmente recomendada para o tratamento da tuberculose, poderia onerar sensivelmente o número de inscritos.
T A B E L A 1

Número de prontuários dos doentes submetidos ao tratamento curto, com inscrição e alta, segundo os Distritos Sanitários. Departamento Regional de Saúde do Vale do Pararba (DRS-3), outubro de 1980 a outubro de 1981 .

\begin{tabular}{lrc}
\hline Distrito Sanitário & \multicolumn{2}{c}{ Prontuários } \\
& No & $\%$ \\
\hline São José dos Campos & 133 & 43,2 \\
Guaratinguetá & 99 & 32,1 \\
Taubaté & 76 & 24,7 \\
\hline Total & 308 & 100,0 \\
\hline
\end{tabular}

\section{Idade e Sexo}

A distribuição da população de estudo, segundo a idade, é mostrada na Tabela 2. As maiores percentagens recaem sobre indivíduos nas idades compreendidas entre $20 \mathrm{e}$ 49 anos. Confirmando dados de trabalhos feitos anteriormente, verifica-se que a tuberculose no nosso meio continua a atingir mais freqüentemente o indivíduo jovem ou na fase de sua maior produtividade.

Trata-se portanto de uma característica que vem se mantendo no correr dos anos e, neste trabalho, a percentagem de doentes pertencentes àquela faixa etária de idade mostra-se maior $(63,7 \%)$ do que as encontradas por Lins de Lima e Nascimento $(58,9 \%)^{8}$ para os anos de 1967,68 e 69 e por Nascimento e Lima $(56,1 \%)^{9}$ entre 1970 e 1974. Para as idades até 19 anos, os dados do trabalho apresentam $17,5 \%$ de indivíduos inscritos, percentagem sensivelmente mais baixa do que aquelas apresentadas nos estudos anteriormente citados: $29,1 \%$ e $29,6 \%$, respectivamente. $O$ inverso ocorre com relação às idades de 50 até 60 anos e mais. A população de estudo foi constituída por $18,8 \%$ de indivíduos nesta idade quando nos dois trabalhos verificou-se uma percentagem de $12,0 \%$ e $12,5 \%$. Isto pode sugerir uma maior proteção às pessoas de baixa idade devido à vacinação pelo BCG intradérmico e o deslocamento do aparecimento da doença para grupos etários mais altos. 
BELLUOMINI, M. \& TAGUSAGAWA, H.K. Sequiência do tratamento de curta duração da tuberculose pulmonar em unidades sanitárias do Vale do Parafba, 1980-1981, Sáo Paulo, Brasil. Rev. Saúde públ, S. Paulo, 18: 466-75, 1984 .

Quanto ao sexo, houve uma leve predominância do sexo masculino sobre o feminino, $57,1 \%$ e $42,9 \%$, respectivamente.

\section{Tipo de Alta}

A sequêencia do tratamento, segundo o tipo de alta concedida aos 308 pacientes inscritos no período estudado é mostrada na Tabela 3.

A alta por cura de $82,5 \%$ ' dos doentes refere-se a altas concedidas até 10 meses e mais de tratamento. Até o 60 mês completo sairam curados $157(61,9 \%)$ doentes, incluindo-se entre eles $24(15,3 \%)$ doentes transferidos de outras instituiçóes, já em tratamen. to com drogas de esquemas de curta duração e que completaram o período e receberam alta a partir do 20 mês de inscrição nas instituiçбes estudadas.

As avaliações dos resultados do tratamento de curta duração no Brasil tem mostrado resultados variáveis. Gerhardt ${ }^{7}$ refere dados iniciais bastante satisfatórios em 90 estabelecimentos de saúde, de 11 capitais brasileiras, com $82,5 \%$ dos doentes com alta por cura ao final do 60 mês de tratamento. Já Almeida ${ }^{1}$, em uma unidade do município de São Paulo, refere a $69,1 \%$ de tratamento completado com sucesso e Souza e Gomes ${ }^{15}$, no Centro de Saúde de Florianópolis, encontraram $64 \%$ de curados.

T A B E L A 2

Distribuição dos doentes segundo sexo e faixa etária. Departamento Regional de Saúde do Vale do Paraíba (DRS-3), outubro de 1980 a outubro de 1981.

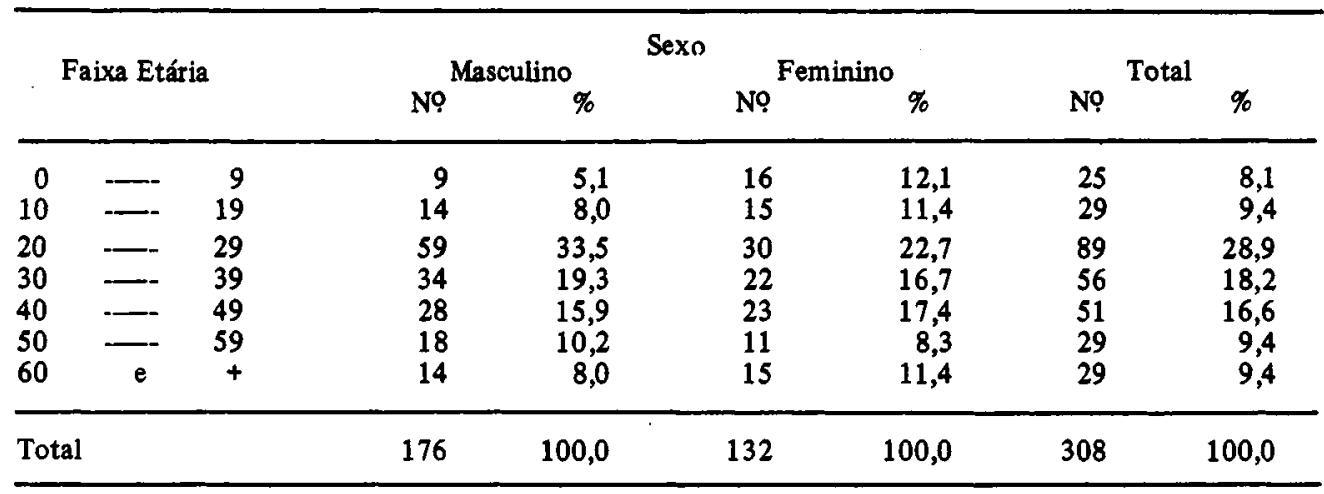

T A B E LA 3

Distribuição dos doentes segundo o tipo de alta. Departamento Regional de Saúde do Vale do Paraíba (DRS-3), outubro de 1980 a outubro de 1981 .

\begin{tabular}{lrr}
\hline Tipo de alta & No & $\%$ \\
\hline Cura & 254 & $\mathbf{8 2 , 5}$ \\
Abandono & 21 & 6,8 \\
Mudança de diagnóstico & 14 & 4,5 \\
Obito & 12 & 3,9 \\
Transferência & 7 & 2,3 \\
\hline Total & 308 & 100,0 \\
\hline
\end{tabular}

Os niveis de eficácia altamente satisfatórios, em geral acima de $90 \%$, encontrados em ensaios epidemiológicos controlados para avaliar o tratamento encurtado da tuberculose, muitas vezes não se repetem em inquéritos feitos entre doentes em tratamento ambulatorial de rotina, visto que o rendimento eprdemiologico dos esquemas recomendados freqüentemente é prejudicado por variáveis comportamentais ou organizacionais.

A alta por abandono foi dada a $6,8 \%$ dos pacientes, no periodo estudado. Do total de 21 abandonos, $6(28,6 \%)$ abandonaram até o 3 ọ mês o que significa que mais de $70 \%$, dos que abandonaram, ultrapassaram 3 
BELLUOMINI, M. \& TAGUSAGAWA, H.K. Seqüência do tratamento de curta duração da tuberculose pulmonar em unidades sanitárias do Vale do Paraíba, 1980-1981, São Paulo, Brasil. Rev. Saúde públ, S. Paulo, 18: 466-75, 1984.

meses de tratamento. Entre o 40 e 50 mês ocorreu o maior número de abandonos. As taxas de abandono apresentadas em alguns trabalhos consultados mostram comporta. mentos bastante diferentes entre os doentes com tratamento de curta duração. Almeida ${ }^{1}$ refere $30,8 \%$ de abandonos entre doentes ambulatoriais estudados; Souza e Gomes ${ }^{15}$ encontraram $17 \%$ e Gerhardt e col. ${ }^{6}$ na revisão de 1.251 prontuários de 90 unidades de saúde em 11 capitais brasileiras mostram uma taxa de $13,1 \%$ de abandono, para todos os doentes em tratamento, inclusive das formas extrapulmonares que foi o grupo que apresentou taxas mais elevadas. Considerando-se apenas os doentes com tuberculose pulmonar, esta taxa cairia para $12,7 \%$. Estas variaçбes no abandono do tratamento pelo doente tuberculoso podem estar vinculadas à parte organizacional dos serviços, já que a atitude da equipe de saúde exerce influência muito grande na manutenção do doente até chegar a alta por $\mathrm{cura}^{3,13}$.

Mudança de diagnóstico foi feita para $4,5 \%$ dos pacientes, em maior número entre o 10 e 20 mês, porém com ocorrências em quase todos os períodos, até mesmo após o 6 ? mês completo de tratamento.

0 óbito ocorreu em $3,9 \%$ dos doentes embora não se possa afirmar que a causa tenha sido a tuberculose, já que a informação obti. da foi a de que 12 doentes faleceram no decorrer do tratamento. Também neste evento a ocorrência foi relativamente constante nos períodos, com maior número até o 19 mês e entre o 2 ? e 30 mês de tratamento. tes.

A transferência foi dada a $2,3 \%$ dos doen.

$\mathrm{Na}$ Figura, são mostradas as al tas concedidas até o 6 mês por períodos de tratamento, e consolidadas a partir do 70 mês, aos doentes inscritos para tratamento curto no periodo estudado.

\section{Esquemas de Tratamento}

A Tabela 4 mostra a distribuição dos doentes segundo o esquema de tratamento adotado. Para 69,8\% dos doentes foi reco-

\section{T A B E L A 4}

Distribuição dos doentes segundo o esquema de tratamento adotado. Departamento de Saúde do Vale do Paraiba (DRS-3), outubro de 1980 a outubro de 1981.

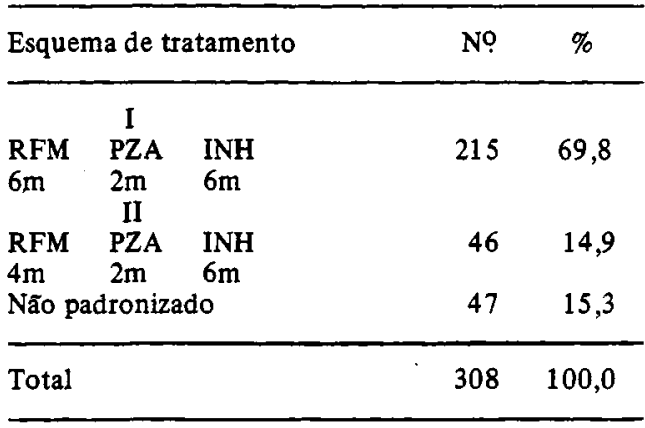

mendado o esquema I padronizado, ou seja, a utilização das 3 drogas (RFM, PZA e INH) por um período de 6 meses, com a suspensão da PZA no 20 mês e a manutenção da RFM e INH até o final do 6 O mês ( 2 PZA, 6 RFM e 6 INH). Para $14,9 \%$ foi recomendado o esquema II, que modificava somente o prazo de manutenção de cada droga (2 PZA, 4 RFM e 6 INH). A alta percentagem de doentes em tratamento com o esquema I e a baixa percentagem daqueles com esquema II sugerem que um número relativamen. te alto de doentes sem confirmação baciloscópica tenham feito tratamento com o esquema I, contrariando as normas que na época determinavam o esquema II para formas pulmonares não confirmadas ${ }^{4}$. A alta percentagem de doentes ${ }^{\vee}(15,3 \%)$ mantidos sob tratamento de curta duração com drogas não constantes dos esquemas padronizados pode ter ocorrido devido à implantação do Convênio INAMPS/Secretaria de Saúde, que se concretizou dentro do período de estudo e, possivelmente, os doentes com esquemas não padronizados seriam aqueles transferidos de serviços conveniados, os quais ainda não teriam adotado as normas recomendadas. Em se tratando de introdução de novas medidas, não se pode deixar de considerar a hipótese de uma certa resistência por parte de alguns profissionais em adotá-las integral e imediatamente. 
BELLUOMINI, M. \& TAGUSAGAWA, H.K. Seqüência do tratamento de curta duração da tuberculose pulmonar em unidades sanitárias do Vale do Paraíba, 1980-1981, Sáo Paulo, Brasil. Rev. Saúde públ, S. Paulo, $18: 466-75,1984$.

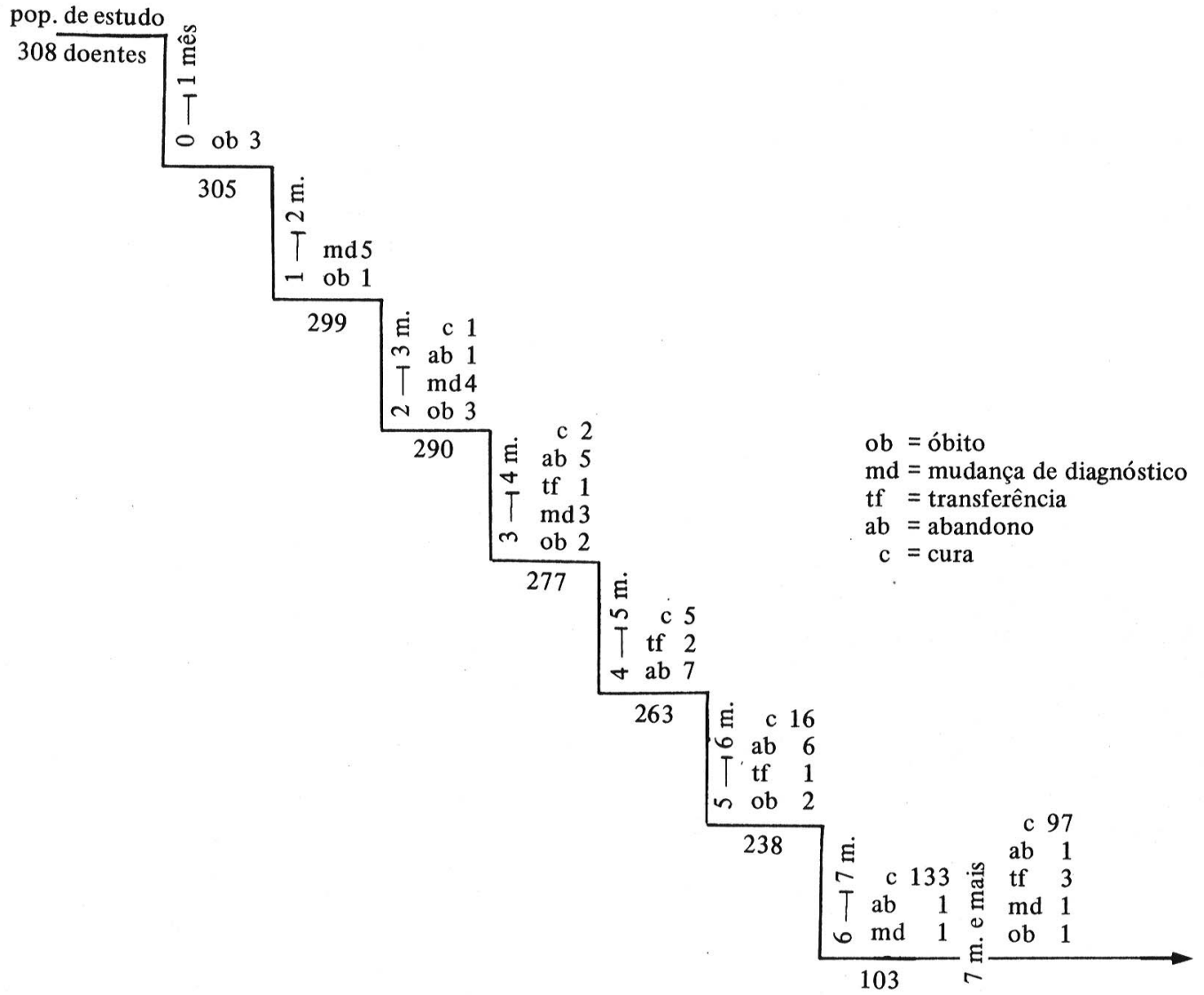

Figura - Motivo das altas concedidas mensalmente até o 69 mês e consolidadas após 7 meses e mais de tratamento da tuberculose pulmonar, aos doentes submetidos ao tratamento encurtado, inscritos e com alta no período de outubro 1980 a outubro 1981 - DRS-3 - São Paulo.

\section{Hospitalização}

Pelos dados apresentados na Tabela 5, observa-se que as recomendações sobre internação vem sendo seguidas na Regional es. tudada. Entre os 254 pacientes com alta cura. da, apenas $2 \%$ dos doentes foram encaminhados para tratamento hospitalar.

Mesmo quando era adotado o tratamento de longa duração, foi comprovada a eficácia do tratamento ambulatorial, conforme relatam os trabalhos de Madras ${ }^{17}$. Um dos argu. mentos mais sérios contra o tratamento ambulatorial é o não isolamento do doente positivo, visto que apresenta um perigo para
T A B E L A 5

Distribuição dos doentes com alta por cura que foram encaminhados para tratamento hospitalar. Departamento Regional de Saúde do Vale do Paraiba (DRS-3), outubro de 1980 a outubro de 1981

\begin{tabular}{lrr}
\hline Hospitalização & Nọ & \multicolumn{1}{c}{ Pacientes } \\
\hline Sim & 5 & 2,0 \\
Não & 249 & 98,0 \\
\hline Total & 254 & 100,0 \\
\hline
\end{tabular}


BELLUOMINI, M. \& TAGUSAGAWA, H.K. Sequiência do tratamento de curta duração da tuberculose pulmonar em unidades sanitárias do Vale do Parafba, 1980-1981, Sáo Paulo, Brasil. Rev. Saúde públ, S. Paulo, $18: 466-75,1984$.

sua familia e pessoas com as quais convive. $O$ tratamento padronizado de curta duração, quando bem administrado, permite uma rápida diminuição da infecciosidade devido ao alto poder bactericida e bacteriostático das drogas recomendadas. Assim, aquela relação de perigo deixa de existir, quando o doente é submetido a uma quimioterapia adequada. Toman $^{16}$ faz uma colocação bastante clara e extremamente oportuna quando diz: "não há justificativa científica nem razóes obje-

T A B E L A 6

Referência a intolerância medicamentosa entre os doentes com alta por cura.

Departamento Regional de Saúde do Vale do Paraiba (DRS-3), outubro de 1980 a outubro de 1981.

\begin{tabular}{lrr}
\hline Intolerância & No Pacientes & $\%$ \\
\hline Sim & 22 & 8,7 \\
Não & 232 & 91,3 \\
\hline Total & 254 & 100,0 \\
\hline
\end{tabular}

tivas para a adoção do tratarnento hospitalar, como política geral para o tratamento da tuberculose. $O$ mais importante é que o pessoal encarregado da quimioterapia, durante todo o tratamento, seja bem orientado na sua responsabilidade de educar o paciente e sua famnlia".

\section{Intolerância Medicamentosa}

As referências à intolerância medicamentosa, no decorrer do tratamento, atingiu $8,7 \%$ dos doentes curados. Gerhardt ${ }^{7}$, tecendo comentários sobre aspectos operativos da quimioterapia de curta duração no Brasil, faz referência a uma experiência que utilizou o mesmo esquema em estudo, ou seja, a rifampicina, a pirazinamida e a isoniazida durante 6 meses, permitindo a verificação da possibilidade desta associação sem maior risco de fenômenos tópicos, com apenas $2 \%$ de necessidade de suspensão das drogas por conseqüência de efeitos colaterais. Souza e Gomes $^{15}$ encontraram entre os doentes em tratamento no Centro de Saúde de Santa Catarina, 6,8\% de referências a in. tolerâncias medicamentosa e Paschoal e col. ${ }^{11}$, no serviço docente - assistencial da Universidade Estadual de Campinas (UNICAMP), não constataram nenhum efeito colateral dependente da quimioterapia.

Importante ressaltar que no presente estudo, em nenhum dos casos houve necessidade de mudança do esquema adotado. Segundo informaçōes colhidas nos Serviços, a suspensão temporária dos medicamentos ou a inclusão de medicação específica a determinados efeitos colaterais foi suficiente para superar o problema.

As reaçðes adversas mais apontadas foram nauseas e vômitos que, juntamente com outras perturbaçzes gástricas, atingiu mais de $70 \%$ dos doentes com intolerância. Outras reaçōes mencionadas foram: amenorréia, perturbaçōes cutâneas e articulares, tontura, síndromes gripais, dormência nas mãos e pés, surdez, deficiência visual.

Tendo em vista os achados anteriormente referidos, a intolerância aqui apresentada é alta. Este é um aspecto que deve ser constantemente observado, pois a toxicidade aos medicamentos pode converter-se em uma das causas de fracasso se năo se tomam as medidas adequadas.

\section{Tempo de Negativação do Escarro}

Dos 254 doentes que tiveram alta por cura no período estudado, 203 (79,9\%) fizeram pesquisa de BK no escarro. Destes, $116(57,1 \%)$ mostraram-se positivos ao exame, ocorrendo uma negativação bacilóscópica de $100 \%$ dos casos após o 40 mês de tratamento. Os resultados encontrados são bastante favoráveis, principalmente quando analisados frente aos achados de Poppe de Figueiredo $^{12}$, em 1965, entre doentes sub. metidos a quimioterapia tríplice standard pelo prazo de um ano, conforme é mostrado na Tabela 7. 
BELLUOMINI, M. \& TAGUSAGAWA, H.K. Seqüência do tratamento de curta duração da tuberculose pulmonar em unidades sanitárias do Vale do Paraíba, 1980-1981, São Paulo, Brasil. Rev. Saúde públ, S. Paulo, 18: 466-75, 1984 .

T A B E L A 7

Negativação baciloscópica em doentes tratados com esquemas tríplices standard durante um ano e com esquemas de curta duração.

\begin{tabular}{llccc}
\hline $\begin{array}{l}\text { Tempo de Negativação } \\
\text { do Escarro (por mês } \\
\text { de tratamento) }\end{array}$ & $\begin{array}{l}\text { Doentes com Tratamento } \\
\text { Standard } \\
\text { Nọ }\end{array}$ & $\begin{array}{c}1965^{*} \\
\%\end{array}$ & $\begin{array}{c}\text { Doentes em Tratamento de Curta } \\
\text { Duração 1980 a 1981** } \\
\text { Nọ }\end{array}$ & $\%$ \\
\hline Após 1 mês & 147 & 60,5 & 96 & 82,7 \\
Após 2 meses & 193 & 79,4 & 112 & 96,5 \\
Após 3 meses & 214 & 88,1 & 114 & 98,2 \\
Após 4 meses & 229 & 94,2 & 116 & - \\
Após 5 meses & 232 & 95,5 & - & - \\
Continuaram positivos ou & 11 & 4,5 & 100,0 \\
recairam & 243 & 100,0 & 116 & 100,0 \\
\hline Total & & & & \\
\hline
\end{tabular}

* H.E.S. Maria e C.S.R. Paula Souza - Rio de Janeiro.

** DRS-3 - São Paulo.

Nota-se uma diferença sensível na queda da positividade dos exames entre os dois grupos, desde o primeiro mês completo de tratamento. Ao final do quarto mês todos os doentes tratados com drogas recomendadas para o tratamento de curta duração mostram-se negativos, o que não ocorreu com aqueles submetidos ao tratamento standard.

Rouillon ${ }^{14}$, disçutindo exaustivamente a atuação das drogas sobre o bacilo, cita vários estudos que mostram o decréscimo da infecciosidade dos doentes quando submetidos a tratamento regular e com esquemas adequados, e Neves ${ }^{10}$ atribui aos esquemas atuais, entre outras, duas relevantes vantagens: extinção rápida da contagiosidade e maior velocidade de negativação do escarro.

\section{CONCLUSÃO}

A adoção de esquemas curtos para tratamento de tuberculose na DRS-3 no período estudado, apresentou desvios das normas com relação ao tempo de tratamento e esquemas utilizados.

Os resultados foram bastante favoráveis quanto a redução da taxa de abandono e de hospitalização, quanto ao rápido decréscimo da positividade dos doentes bacilíferos sob tratamento e também quanto à percentagem de altas por cura. Essas condiçôes permitem concluir que houve um bom desempenho da equipe treinada para o atendimento do doente nas Unidades trabalhadas, e que o tratamento encurtado da tuberculose pulmonar bem conduzido, proporciona excelentes resultados não só sob o ponto de vista operacional, mas principalmente sob o ponto de vista epidemiológico, pela eliminação a curto prazo das fontes de infecção. 
BELLUOMINI, M. \& TAGUSAGAWA, H.K. Sequiência do tratamento de curta duração da tuberculose pulmonar em unidades sanitárias do Vale do Paraiba, 1980-1981, São Paulo, Brasil. Rev. Saúde públ, S. Paulo, 18: 466-75, 1984 .

BELLUOMINI, M. \& TAGUSAGAWA, H. K. [Evaluation of short-term chemotherapy for pulmonary tuberculosis in the Health Institutions of the Vale do Paralba, 1980-1981, S. Pau1o, Brazil.] Rev. Saúde públ., S. Paulo, 18: 466-75,1984.

ABSTRACT: The results of the short-term chemotherapy for pulmonary tuberculosis performed on patients who were admitted to the Health Clinics of one of the S. Paulo State's Health Divisions and who completed the treatment during the period from October 1980 to October 1981 are shown. Such aspects of the records as Health Districts, patient's age and sex, success and regimen of treatment, hospitalization, drug toxicity and smear negativity period were analysed. The findings confirm the effectiveness of short-term chemotherapy under program conditions as corroborated by its acceptability to the patients, the success achieved and the benefits in the decrease of the number of admissions to hospital and in the reduction of the sources of infection within a shorter period.

UNITERMS: Tuberculosis, pulmonary, therapy. Ambulatory care. Tuberculosis, chemotherapy.

\section{REFERENCIAS BIBLIOGRÁFICAS}

1. ALMEIDA, M.M.M.B. Contribuiçăo ao processo de avaliaçăo do tratamento da tuberculose; estudo realizado em um Centro de Saúde de Săo Paulo. Sáo Paulo, 1981. [Dissertação de Mestrado - Faculdade de Saúde Pública USP]

2. BELLUOMINI, M. Conhecimento sobre alguns aspectos da tuberculose pulmonar entre doentes internados em hospitais especializados. Saro Paulo, 1981. [Tese de Doutoramento - Faculdade de Saúde Priblica USP]

3. CABRAL, J. The importance of motivation in fully supervised ambulatory treatment. Bull. int. Un. Tuberc., 47: 84-9, 1972.

4. DIVISÃo NACIONAL DE PNEUMOLOGIA SANITARIA. Manual de normas para o controle da tuberculose. Rio de Janeiro, 1979.

5. FOX, W. \& MTCHISON, D.A. Short-course chemotherapy for pulmonary tuberculosis. Amer. Rev. resp. Dis, 111 : 325-53, 1975.

6. GERHARDT, G. et al. Aspectos operativos na quimioterapia de curta duração no programa de tuberculose no Brasil. [Apresentado no III Seminário Regional de Quimioterapia, Washington, D. C., 1979]

7. GERHARDT, G, et al. Resultados iniciais do tratamento de curta duração em condições de rotina nos Serviços de Saúde no Brasil. [Apresentado no XXII Congresso Internacional de Pneumologia e Tísiologia, Buenos Aytes, 1982]
8. LINS DE LIMA, L. \& NASCIMENTO, E.A. do Algumas informações sobre a tuberculose no Brasil de 1960-1969. Rev. Div. nac. Tuberc., 15: 549, 1971.

9. NASCIMENTO, E.A. do \& LIMA, R. L. Algumas informaçōes sobre a tuberculose no Brasil - 1970-1974. Rev. Div. Nac. Tuberc., 20:119-39, 1976.

10. NEVES, J.S. $O$ tratamento em massa da tuberculose. Rev. Div. nac. Pneum. sanit., 22: 115-25, 1978.

11. PASCHOAL, I. et al. Análise do resultado da quimioterapia ambulatorial de 123 pacientes tratados em Serviço docente Assistencial. J. Pneumol., 8(supl.): 148-9, 1982.

12. POPPE DE FIGUEIREDO, F. A curva de negativação baciloscópica no controle de tratamento dos casos novos de tuberculose pulmonar. Rev. Serv. nac. Tuberc., 11: $338-49,1967$.

13. ROUILLON, A. Problems in organising effective ambulatory treatment of tuberculosis patients. Bull int. Un. Tuberc., 47:43-83, 1972.

14. ROUILLON, A. et al. Transmission of tubercle bacilli: the effects of chemotherapy. Tubercle, 57: 275-99, 1976.

15. SOUZA, R. P. \& GOMES, C. Tratamento de curta duração da tuberculose no Centro de Saúde de Florianópolis, SC. J. Pneumol, 8(supl.): 137, 1982. 
BELLUOMINI, M. \& TAGUSAGAWA, H.K. Sequiência do tratamento de curta duração da tuberculose pulmonar em unidades sanitárias do Vale do Paraíba, 1980-1981, São Paulo, Brasil. Rev. Saúde públ, S. Paulo, 18: 466-75, 1984.

16. TOMAN, K. Tuberculosis: detection de casos e quimioterapia. Washington, D.C., Organización Panamericana de la Salud, 1980. p. 242-7. (OPAS-Publ. cient., 392).

17. TUBERCULOSIS CHEMOTHERAPY CENTRE - MADRAS. A concurrent comparison of homes and Sanatorium treatment of pulmonary tuberculosis in Shouth India. Bull. Wld Hlth Org., 21 : 51-114, 1959.
18. WAKSMAN, S.A. A vitória sobre a tuberculose. São Paulo, Cultrix, 1966.

Recebido para publicação em 11/07/1984. Aprovado para publicação em 04/10/1984. 